\title{
Transcription factor HBP1 is a direct anti-cancer target of transcription factor FOX01 in invasive oral cancer
}

\author{
Chien-Yi Chan ${ }^{1}$, Shih-Yi Huang ${ }^{2}$, Jim Jinn-Chyuan Sheu ${ }^{3,4}$, Mendel M. Roth ${ }^{5}$, I-Tai \\ Chou $^{1}$, Chia-Hsien Lien ${ }^{1}$, Ming-Fen Lee ${ }^{6, *}$, Chun-Yin Huang ${ }^{1,4, *}$ \\ ${ }^{1}$ Department of Nutrition, China Medical University, Taichung, Taiwan \\ ${ }^{2}$ School of Nutrition and Health Sciences, Taipei Medical University, Taipei, Taiwan \\ ${ }^{3}$ Institute of Biomedical Sciences, National Sun Yatsen University, Kaohsiung, Taiwan \\ ${ }^{4}$ Department of Health and Nutrition Biotechnology, Asia University, Taichung, Taiwan \\ ${ }^{5}$ GB Lifesciences, San Diego, CA, USA \\ ${ }^{6}$ Department of Nutrition and Health Sciences, Chang Jung Christian University, Tainan, Taiwan \\ *These authors contributed equally to this work
}

Correspondence to: Chun-Yin Huang, email: chuang@mail.cmu.edu.tw

Ming-Fen Lee, email: leemf@mail.cjcu.edu.tw, summerblueair@yahoo.com

Keywords: FOXO1, HBP1, oral cancer

Received: September 16, $2016 \quad$ Accepted: January 07, $2017 \quad$ Published: January 14, 2017

\section{ABSTRACT}

Either FOXO1 or HBP1 transcription factor is a downstream effector of the PI3K/ Akt pathway and associated with tumorigenesis. However, the relationship between FOXO1 and HBP1 in oral cancer remains unclear. Analysis of $\mathbf{3 0}$ oral tumor specimens revealed that mean mRNA levels of both FOXO1 and HBP1 in non-invasive and invasive oral tumors were found to be significantly lower than that of the control tissues, and the status of low FOXO1 and HBP1 ( $<0.3$ fold of the control) was associated with invasiveness of oral tumors. To investigate if HBP1 is a direct transcription target of FOXO1, we searched potential FOXO1 binding sites in the HBP1 promoter using the MAPPER Search Engine, and two putative FOXO1 binding sites located in the HBP1 promoter -132 to -125 bp and -343 to -336 bp were predicted. These binding sites were then confirmed by both reporter gene assays and the in cellulo ChIP assay. In addition, Akt activity manipulated by PI3K inhibitor LY294002 or Akt mutants was shown to negatively affect FOXO1-mediated HBP1 promoter activation and gene expression. Last, the biological significance of the FOX01-HBP1 axis in oral cancer malignancy was evaluated in cell growth, colony formation, and invasiveness. The results indicated that HBP1 knockdown potently promoted malignant phenotypes of oral cancer and the suppressive effect of FOXO1 on cell growth, colony formation, and invasion was alleviated upon HBP1 knockdown in invasive oral cancer cells. Taken together, our data provide evidence for HBP1 as a direct downstream target of FOXO1 in oral cancer malignancy.

\section{INTRODUCTION}

The forkhead box O (FOXO) transcription factor family shares a conserved 'forkhead box' DNA-binding domain and is consisted of four members in mammals: FOXO1, FOXO3a, FOXO4 and FOXO6 [1]. FOXO factors are involved in a wide range of biological processes, including cell cycle arrest, apoptosis, DNA repair, glucose metabolism, oxidative stress resistance and longevity [1]. The biological activity of FOXO factors is mostly dependent on the post-translational modification of phosphorylation, acetylation, or ubiquitination, thereby determining their intracellular trafficking [2]. Among FOXO factors, increased p-FOXO1 or decreased FOXO1 expression is often associated with tumorigenesis [3]. FOXO1 may exert its tumor suppression function through its transcription-dependent expression of growth arrest and apoptotic-related genes, including p15, p19, NOXA, FasL, TRAIL, and Bim [4-6]. The promoters of these genes comprise the FOXO-recognized element with the consensus 
sequence T/C-G/A-A-A-A-C-A-A [7]. Together, FOXO1 appears to be a potential therapeutic target of anticancer reagents.

HMG box-containing protein 1 (HBP1) is a regulator of cell cycle exit and cell differentiation [8]. Overexpression of HBP1 leads to an inhibition of the G1-S phase transition [9]. Loss-of-function HBP1 variants have been isolated in myeloid leukemia and breast cancers $[10,11]$. Conversely, ectopic or chemical-induced expression of HBP1 results in growth arrest, apoptosis, or differentiation in cancer cell lines, including oral cancer [12-15]. Together, these findings suggest a role of HBP1 in tumor suppression. HBP1 exerts its repression function through an HMG box DNA-binding domain and an AXH transcriptional repression domain. Genes down-regulated by HBP1 include $p 47 p h o x, N-m y c, c-m y c, C C N D 1$, and $\operatorname{MIF}[9,14,16,17]$.

Studies of HBP1 have been focused on the finding of its transcription downstream targets. How the $H B P I$ promoter is regulated remains unclear. In a previous study, we demonstrated that HBP1 is a downstream effector of the EGFR (epidermal growth factor receptor))/ Akt pathway in oral cancer [15]. Subsequently, a recent report illustrated that HBP1 is direct target of growth factors-mediated PI3K/Akt/FOXO pathway in various types of cancer [18]; however, whether or not FOXO1 regulates HBP1 expression in oral cancer remains unclear. In this study, we reported that both HBP1 and FOXO1 were down-regulated in a subset of oral tumor specimen. Ectopic expression of FOXO1 led to increased $\mathrm{HBP} 1$ promoter activity and HBP1 expression in oral cancer cells. Indeed, FOXO1-influencing factors, such as Akt activity, also accordingly affect $H B P 1$ promoter activity and HBP1 expression. The direct binding of FOXO1 onto the $H B P 1$ promoter was evidenced by the identification of two putative FOXO1 consensus sites in its cis-acting region. Furthermore, the biological significance of FOXO1-mediated HBP1 expression was demonstrated by the experiments of colony formation and cell invasion in oral cancer cells. Together, the current study provided evidence that FOXO1 and HBP1 function coordinately as tumor suppressors in invasive oral cancer.

\section{RESULTS}

\section{Low FOXO1/low HBP1 expression predicts invasiveness of oral cancer}

FOXOs transcription activity is tightly regulated by the Akt signaling [19]. Previously, we demonstrated that HBP1 functions as a downstream effector of the EGFR/Akt signaling pathway in oral cancer [15]. Both FOXOs and HBP1 are negatively regulated by Akt. FOXOs are directly modified by Akt phosphorylation for subsequent degradation; however, how HBP1 expression is down-regulated by Akt remains unclear in oral cancer. Using the MAPPER Search Engine to predict the putative transcription factor binding sites (TFBSs) in the $H B P 1$ promoter, we identified several potential FOXO1 binding sites in the proximal region of the $H B P 1$ promoter. Therefore, we hypothesized that FOXOs may play a crucial role in Akt-mediated suppression of HBP1 expression. Hence, the current study is aimed at investigating the putative transcriptional regulation of HBP1 by FOXO1 and, collectively, the biological significance of FOXO1 and HBP1 in oral cancer.

First, we examined the association of FOXO1 and HBP1 with oral tumorigenicity by comparing the mRNA levels of FOXO1 and HBP1 from 30 oral tumor specimens obtained from China Medical University Hospital (Taichung, Taiwan) with those of nine normal tissues. The mean mRNA level of either HBP1 or FOXO1 in noninvasive and invasive oral tumors (lymph node metastasis) was significantly lower than those of control normal tissues (Figure 1A). Reduced expression of both FOXO1 and HBP1 was also found in two tongue squamous cell carcinoma datasets in the Oncomine database (Supplementary Figure 1). Further analysis indicated a correlation of a low-HBP1 and FOXO1 status ( $<0.3$ fold) with the aggressiveness of oral tumors (Figure 1B). Indeed, when we tested the correlation between HBP1 and FOXO1 expression in aggressive oral cancer specimens, we found that HBP1 mRNA levels were positively correlated with FOXO1 mRNA levels in invasive oral cancer (Figure 1C). Furthermore, both FOXO1 and HBP1 mRNA levels were significantly lower in three EGFR over-expressing oral cancer cells tested than the normal human oral keratinocyte (HOK) (Figure 1D). These clinical data suggest that a combination of low HBP1 and low FOXO1 status might determine oral cancer malignancy, and that HBP1 and FOXO1 expression might be coordinately regulated.

\section{FOXO1 transcriptionally induces HBP1 expression}

To illustrate FOXO1 regulation of HBP1 expression in oral cancer, first, we examined if HBP1 expression levels are in concert with FOXO1 levels. Ectopic expression of FOXO1 in HSC-3 cells with low endogenous FOXO1 levels (Figure 2A) resulted in increased expression of HBP1 protein and mRNA (Figure 2B). Similarly, knockdown of FOXO1 expression with FOXO1-specific siRNA led to reduced HBP1 expression in TW206 oral cancer cells with high level of endogenous FOXO1 (Figure 2C). These data suggest that HBP1 might be transcriptionally regulated by FOXO1. Indeed, ectopic expression of FOXO1 cDNA ( 0.2 and $0.4 \mu \mathrm{g})$ in $293 \mathrm{~T}$ cells for $24 \mathrm{~h}$ enhanced the transcriptional activity of a $2-\mathrm{kb}$ HBP 1 promoter by 5 and 7 folds, respectively (Figure 2D). In addition, FOXO1 exhibited stronger activation of the 2-kb HBP1 promoter than FOXO3a (Figure 2E) although FOXO1 and FOXO3a share highly similar sequence [20]. 
FOXO1-induced activation of the 2-kb $H B P 1$ promoter was also observed in HSC-3 oral cancer cells, and the induction was abolished when co-transfected with FOXO1-specific siRNA (Figure 2F). Taken together, these results indicate that FOXO1 is able to induce HBP1 expression through transcription.

\section{FOXO1 activity is essential for the FOXO1-mediated HBP1 expression}

The EGFR/PI3K/Akt signaling is often up-regulated in malignant oral cancer $[21,22]$. Transcriptional activity of FOXO1 can be suppressed through Akt-mediated post-translational modifications [19]. In previous studies, we demonstrated that activation of Akt leads to downregulation of HBP1 expression (Figure 4A in [15]), and inhibition of Akt phosphorylation by LY294002, a chemical inhibitor of PI3K, results in increased protein level of FOXO1 (Figure 4F in [23]). Hence, we studied whether FOXO1 activation of the HBP1 promoter is also under the control of the upstream regulator Akt in the HSC-3 oral cancer cell line with an aberrant activation of the EGFR/PI3K pathway [23]. Indeed, administration of LY294002 $(20 \mu \mathrm{M})$ to HSC-3 cells potently suppressed Akt
A

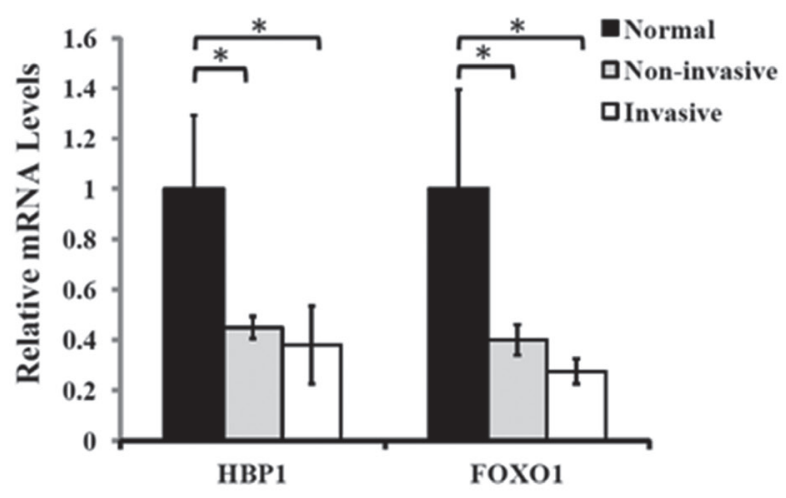

C

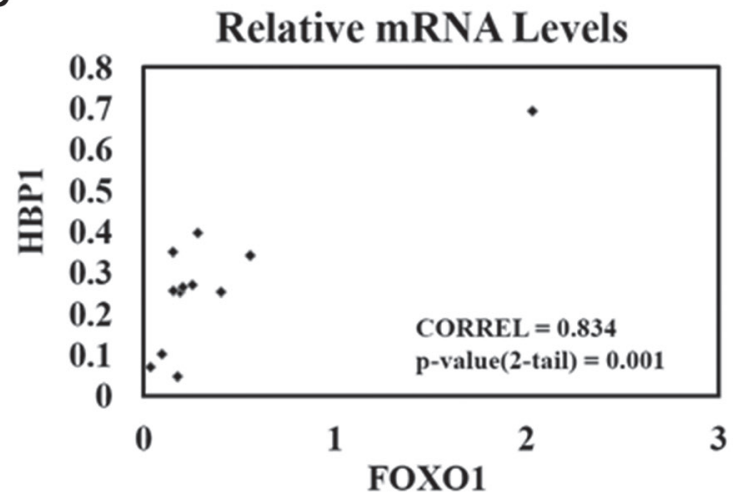

B

\begin{tabular}{|c|c|c|c|}
\hline $\begin{array}{c}\text { Expression of } \\
\text { FOXO1 and } \\
\text { HBP1 }\end{array}$ & Non-invasive & Invasive & Total \\
\hline $\begin{array}{c}\text { Low FOXO1 } \\
\text { and low } \\
\text { HBP1 }\end{array}$ & 2 & 7 & 9 \\
\hline $\begin{array}{c}\text { High FOXO1 } \\
\text { and/or high } \\
\text { HBP1 }\end{array}$ & 16 & 5 & 21 \\
\hline Total & 18 & 12 & 30 \\
\hline
\end{tabular}

2X2 Correlation Table

The status of FOXO1 and HBP1 vs. invasiveness Fisher's Exact Test: p-value $=\mathbf{0 . 0 1 2 5}$

Low FOXO1 and low HBP1: $<0.3$ fold

D

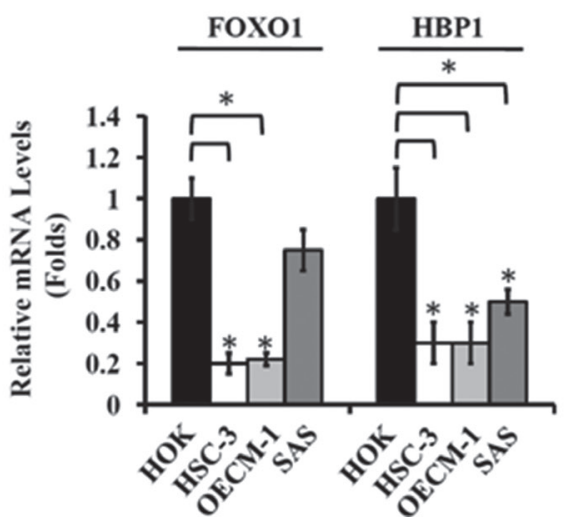

Figure 1: Coordinate down-regulation of FOXO1 and HBP1 in oral cancer. (A) Quantitative analysis of FOXO1 and HBP1 expression levels in oral tumor specimens. RT-qPCR was used to measure FOXO1 and HBP1 mRNA levels of 30 oral tumor specimens. All 30 tumor specimens were divided into two groups, non-invasive $(n=18, \mathrm{pN}=0$, no metastasis $)$ and invasive $(n=12$, pN $>0$, lymph node metastasis). The mean mRNA levels of FOXO1 and HBP1 from 9 adjacent normal tissue specimens were set as 1 with GAPDH as an internal control. The mean mRNA levels of FOXO1 and HBP1 from non-invasive and invasive oral tumor specimens were significantly lower than that of control, normal tissues. Values were expressed as mean \pm S.E.M. $(* p<0.05)$. (B) Association of the FOXO1/HBP1 expression status and invasiveness. Tumors with low FOXO1 and HBP1 $(<0.3$-fold of normal $)$ expression are associated with invasiveness in oral cancer specimens. The $2 \times 2$ correlation table and Fisher's exact test were used, with a significant two-sided value, $p=0.0125$. (C) Correlation between FOXO1 and HBP1 expression in invasive oral tumor specimens. The relative mRNA levels of FOXO1 and HBP1 from 12 invasive oral specimens were under Pearson correlation examination with a coefficient of correlation $0.835(p=0.001)$. (D) The mRNA levels of FOXO1 and HBP1 in oral cancer cell lines. Total RNA were extracted from similar cell density of HOK human oral keratinocytes, and HSC-3, OECM-1, and SAS oral cancer cell lines and subjected to RT-qPCR analysis for both FOXO1 and HBP1 expression. The mRNA levels of FOXO1 and HBP1 were expressed as relative to $18 \mathrm{~S}$, an internal reference $(* p<0.05$ as compared with HOK). 
and FOXO1 phosphorylation with a concomitant increase in HBP1 protein expression (Figure 3A). LY294002 also caused increased mRNA level of HBP1 in HSC-3 cells (Figure 3B). In addition, the reporter gene assay revealed that LY294002 enhanced the FOXO1-mediated HBP1 promoter activity in a dose-dependent fashion (Figure 3C). Then we tested the direct effect of Akt on FOXO1mediated HBP1 expression. Overexpression of either wild-type (Akt1) or constitutively active Akt1 (MyrAkt1) potently abolished FOXO1-induced activation of the 2-kb HBPl promoter (upper panel, Figure 3D) and the expression of $H B P 1$ protein (lower panel, Figure 3D). However, the Akt mutant (Akt1 T308A/S473A) had no significant effect on FOXO1-mediated activation of HBP1 promoter and protein expression (Figure 3D). These results suggest that Akt phosphorylation of FOXO1 might modulate the transcriptional activity of the HBP1 promoter. We further employed FOXO1AAA, a constitutively active FOXO1 mutant, with triple and S319A, [24] to test this hypothesis. FOXO1-AAA mutation on the Akt phosphorylation sites, T24A, S256A,

overexpression showed a stronger enhancement effect on the promoter activity of $H B P 1$ than that of wild-type FOXO1 overexpression in HEK 293T cells (Figure 3E). In addition, the result from the DNA-binding defective FOXO1 (H215R) [24] indicated that this domain is also crucial for the full activation of FOXO1 on the $H B P 1$ promoter (Figure 3E). These data demonstrated that both the protein activity and its DNA-binding ability of FOXO1 are crucial for the FOXO1-mediated HBP1 activation. In addition, the FOXO1-HBP1 axis is a crucial downstream effector of the EGFR/PI3K/Akt signaling pathway in oral cancer.

\section{Identification of the FOXO1 response elements in the $H B P 1$ promoter}

Next, we studied if the regulation of FOXO1 in the transcription of HBP1 is through direct binding to the HBP1 promoter. Three putative FOXO1 binding sites with a core recognition sequence $(\mathrm{T} / \mathrm{C} / \mathrm{G})(\mathrm{G} / \mathrm{A} / \mathrm{T}) \mathrm{AAA}(\mathrm{C} / \mathrm{A})$ A or TT(G/A)TTT(G/A)(G/C)[24, 25] were found on the

C

A

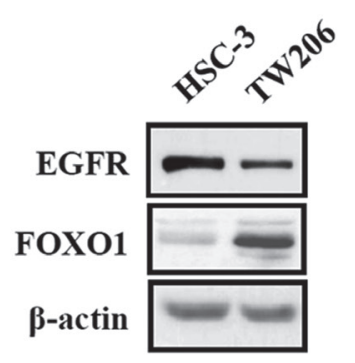

B

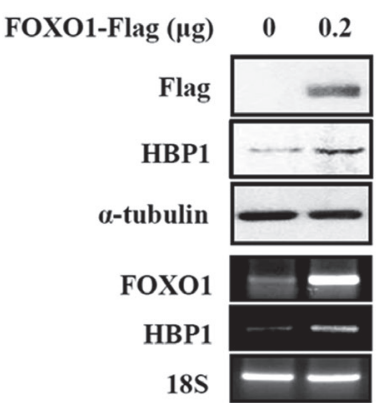

E
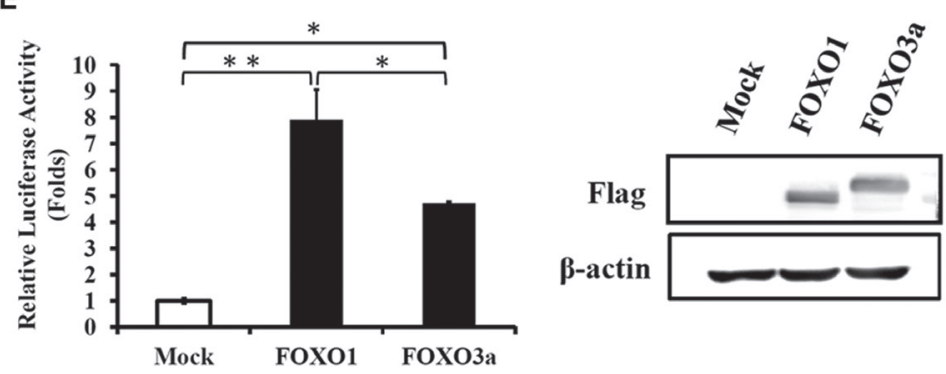

FOXO1 siRNA

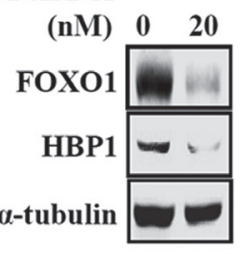

D

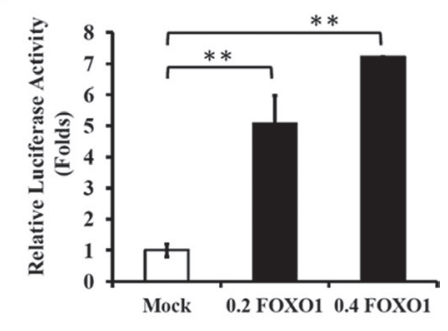

$\mathbf{F}$

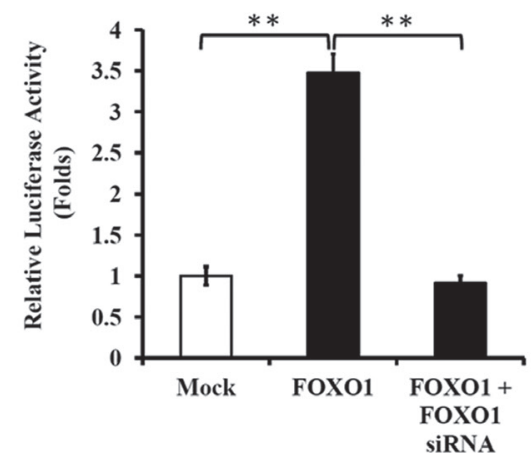

Figure 2: FOXO1 induces HBP1 gene expression in oral cancer. (A) The protein levels of EGFR and FOXO1 in HSC-3 and TW206 oral cancer cell lines. Cell lysates from these two cell lines were isolated and then subjected to Western blotting analysis for the detection of endogenous EGFR and FOXO1. (B) HSC-3 cells were transfected with pcDNA3-FOXO1-Flag $(0.2 \mu \mathrm{g})$ or empty vector pcDNA3 for $24 \mathrm{~h}$, and then cell lysates were collected for analysis of the protein levels of FOXO1-Flag, HBP1 and $\alpha$-tubulin by Western blotting (upper panel). Total RNA extracted from $24 \mathrm{~h}$ of FOXO1-Flag-transfected HSC-3 cell was subjected to quantification of FOXO1 and HBP1 mRNA expression by RT-PCR with $18 \mathrm{~S}$ as internal control (lower panel). (C) Expression levels of FOXO1, HBP1, and $\alpha$-tubulin were examined by Western blotting in TW206 oral cancer cells with FOXO1-specific siRNA transfection for $24 \mathrm{~h}$. (D-F) Effect of FOXO1 on $H B P 1$ promoter. HEK-293T cells seeded in a 24 -well plate were transfected with (D) increasing amount of FOXO1 (0, 0.2, $0.4 \mu \mathrm{g})$, (E) FOXO1 or FOXO3a $(0.4 \mu \mathrm{g})$ along with a $2-\mathrm{kb} H B P 1$ promoter-luciferase construct $(0.4 \mu \mathrm{g})$ for $24 \mathrm{~h}$. (E, upper panel) Luciferase intensities were measured and normalized to $\beta$-galactosidase activities $(* p<0.05 ; * p<0.01)$, and (E, lower panel) expression levels of transfected Flag-FOXO1 and Flag-FOXO3a cDNA were detected using anti-Flag antibody by Western blotting analysis. (F) HSC-3 cells in a 24-well plate were transfected with FOXO1 $(0.2 \mu \mathrm{g})$ and FOXO1 siRNA $(20 \mathrm{nM})$ together with a 2-kb HBP1 promoter-luciferase construct $(0.4 \mu \mathrm{g})$ for $24 \mathrm{~h}$. Luciferase intensities were measured and normalized to $\beta$-galactosidase activities $(* * p<0.01)$. 
A
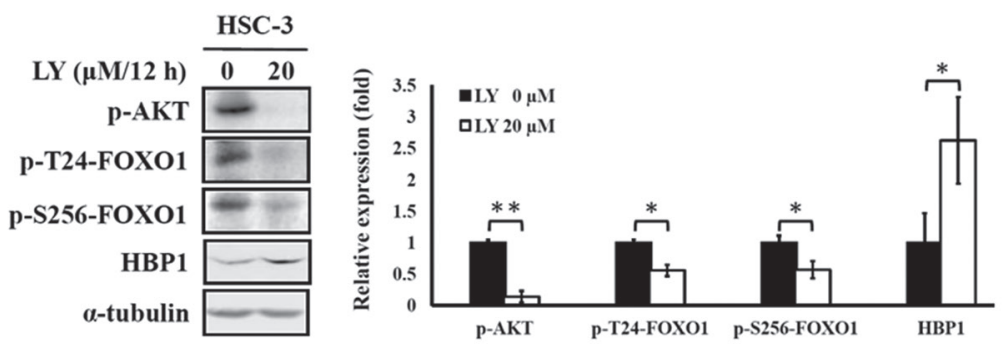

B

C
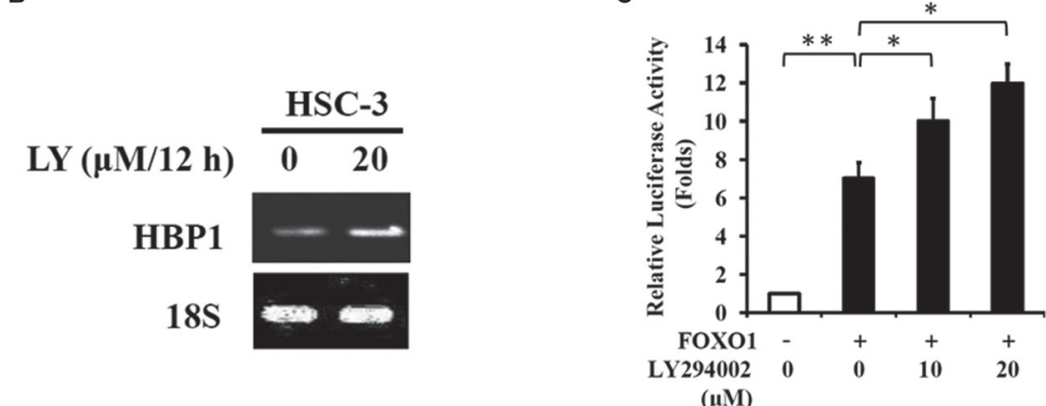

D

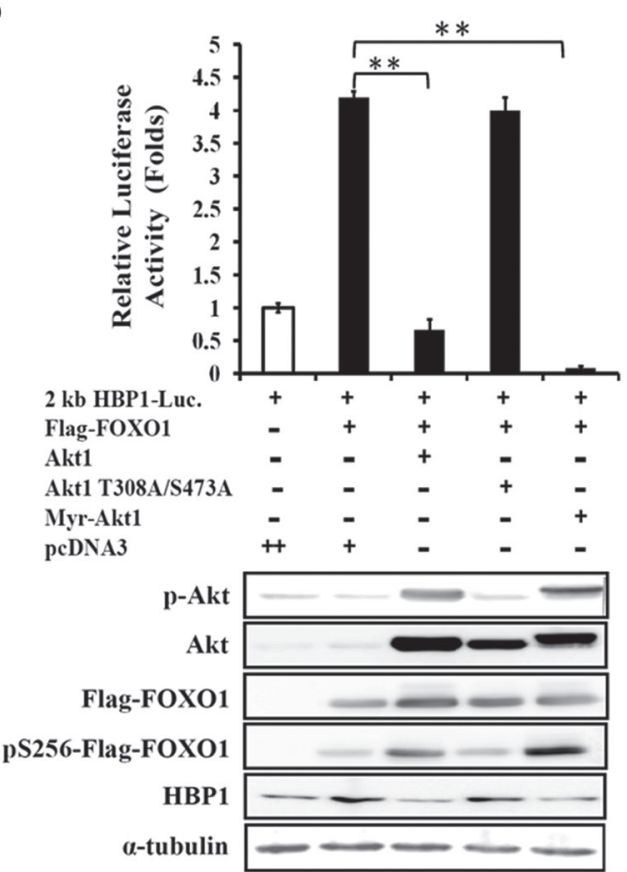

E

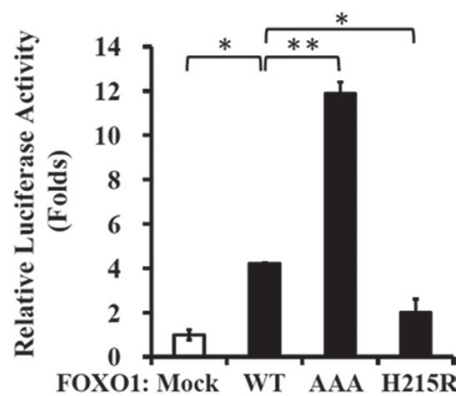

Figure 3: HBP1 expression is regulated by FOXO1 activity. (A-C) Effect of LY294002 on FOXO1-mediated HBP1 expression. (A) HSC-3 cells were treated with LY294002 $(20 \mu \mathrm{M})$ up to $12 \mathrm{~h}$, followed by detection of p-Akt, p-FOXO1, HBP1, and $\alpha$-tubulin expression by Western blotting analysis. One representative experiment out of three independent experiments is shown. Values are mean $\pm \mathrm{SD}$ $\left({ }^{*} p<0.05\right.$ and ${ }^{* *} p<0.01$ as compared with vehicle alone). (B) Total RNA isolated from LY294002-treated HSC-3 cells was subjected to RT-PCR analysis for the measurement of HBP1 mRNA level with 18S as an internal control. (C) HEK-293T cells were transfected with a 2-kb $H B P 1$ promoter-luciferase construct, FOXO1 expressing vector, and $\beta$-galactosidase plasmid for $24 \mathrm{~h}$, followed by treatment of increasing concentration of LY294002 $(0-20 \mu \mathrm{M})$ for additional $24 \mathrm{~h}$. Luciferase intensities were measured and normalized to $\beta$-galactosidase activities $\left({ }^{*} p<0.05 ; * *<0.01\right)$. (D) Effect of Akt activity on FOXO1-mediated HBP1 expression. A 2-kb HBP1 promoter-luciferase construct $(0.2 \mu \mathrm{g})$, a $\beta$-galactosidase plasmid $(0.1 \mu \mathrm{g})$, and FOXO1-Flag cDNA $(0.2 \mu \mathrm{g})$ were co-transfected with $0.2 \mu \mathrm{g}$ of pcDNA3 empty vector, Akt1, Akt1 T308A/S473A mutant, or constitutively active Myr-Akt1 vector into HEK-293T cells cultured in a 24-well plate. After $48 \mathrm{~h}$ of incubation, (D, upper panel) luciferase intensities were measured and normalized to $\beta$-galactosidase activities $(* * p<0.01$ ), and (D, lower panel) expression levels of p-Akt, Akt, Flag-FOXO1, p-Flag-FOXO1, and HBP1 were measured by Western blotting with $\alpha$-tubulin as an internal control. (E) Effect of different FOXO1 domain on HBP1 promoter activity. A 2-kb HBP1 promoter-luciferase construct and a $\beta$-galactosidase plasmid were co-transfected with a pcDNA3 empty vector, FOXO1, FOXO1-H215R or constitutively active FOXO1-AAA vector $(0.2 \mu \mathrm{g})$ into HEK-293T cells. After $48 \mathrm{~h}$ of transfection, luciferase intensities were measured and normalized to $\beta$-galactosidase activities $\left({ }^{*} p<0.05 ; * * p<0.01\right)$. 
upstream region -132 to $-125,-343$ to -336 , and -380 to -373 bp from the HBP1 transcription start site (denoted as F1, F2, and F3, respectively) (Figure 4A). To test the effect of these potential FOXO1 binding sites, first, we cotransfected a pGL3-based luciferase reporter gene carrying a $0.2 \mathrm{~kb}, 0.5 \mathrm{~kb}$, or $2 \mathrm{~kb} H B P 1$ promoter region along with various FOXO1 cDNA clones into HEK-293T cells, and we found that FOXO1 was able to enhance the $H B P 1$ promoter activity of all three regions with a maximal activation for the $0.5 \mathrm{~kb}$ promoter (Figure 4B). This result suggests that FOXO1 binding sites might be located within the proximal region of the $H B P 1$ promoter. To further differentiate and pinpoint the significance of these three potential FOXO1 sites, we carried out a serial deletion on F1, F2, and/or F3 in the $0.5 \mathrm{~kb}$ HBP1-luciferase reporter plasmid as depicted in Figure 4C. Loss of F1, F2, or F3 diminished FOXO1mediated activation, and the deletion of both F1 and F2 completely abolished FOXO1 activation of the HBP1

A

HBP1 promoter region

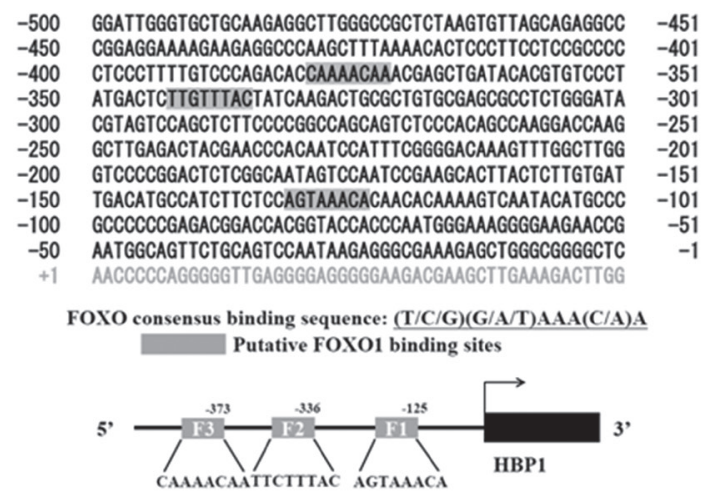

C

0.5 kB HBP1-Luc

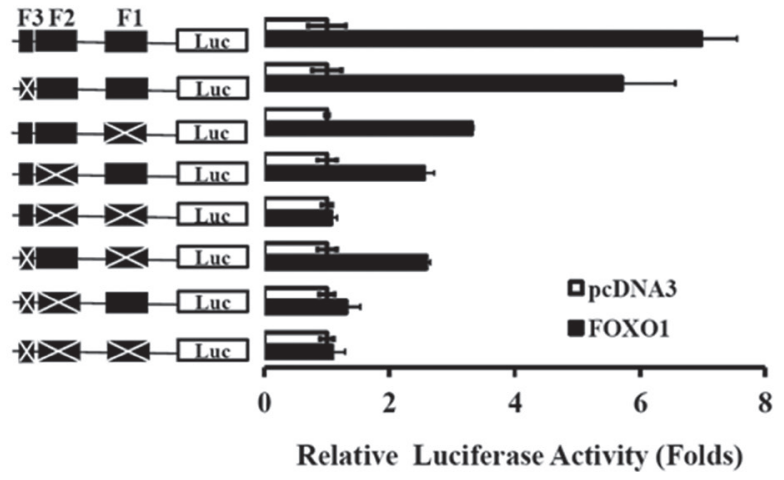

promoter (Figure 4C). Next we examined if FOXO3a also mediated the activity of the $H B P 1$ promoter through these binding sites. As shown in Figure 4D, F2 box is crucial for FOXO3a-mediated activation of the $H B P 1$ promoter while both F1 and F2 boxes are important for FOXO1.

Lastly, to investigate whether FOXO1 binds to the human $H B P 1$ promoter with the context of native human chromatin, chromatin immunoprecipitation (ChIP) assays were performed in HEK-293 cells [24]. Figure 5A depicted the $151 \mathrm{bp}$ and $223 \mathrm{bp}$ primer sets spanning the F1 and F2 boxes in the human $H B P 1$ promoter starting at $-125 \mathrm{bp}$ and -336 bp, respectively, for the PCR analysis. FOXO1Flag overexpression protein formed a complex with either region of the endogenous human $H B P 1$ promoter, whereas the $H B P 1$ promoter binding signal was barely detected in the negative control, GAPDH immunoprecipitation (Figure 5B). In contrast, FOXO1- $\triangle \mathrm{DB}$-Flag, lacking the DNA binding domain, obviously diminished the binding

B

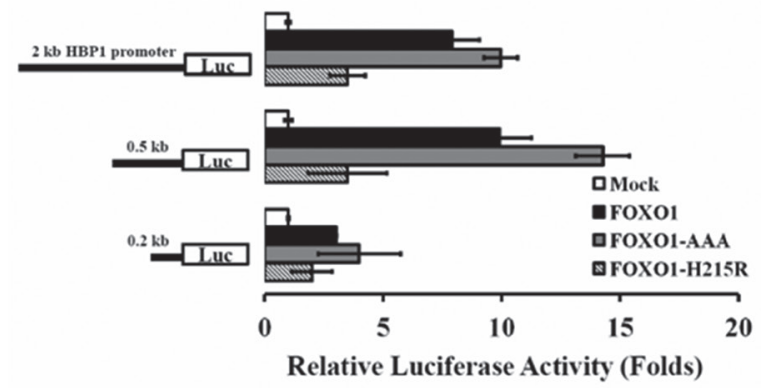

D

0.5 kB HBP1-Luc

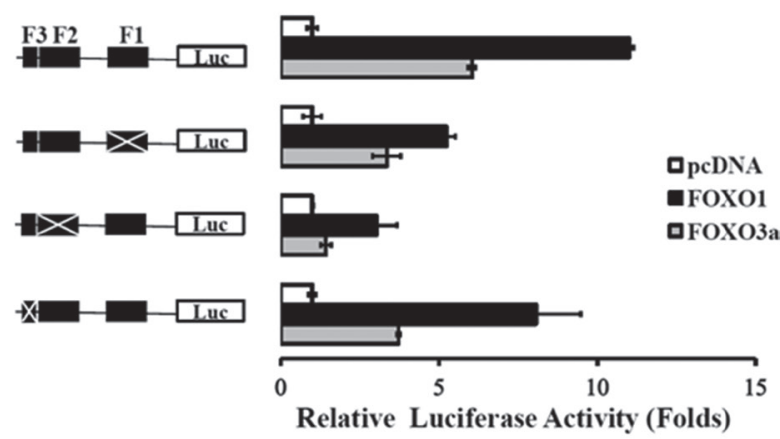

Figure 4: Identification of FOXO1 response elements in the HBP1 promoter. (A) Schematic diagram of the $H B P 1$ proximal promoter containing three potential FOXO1 binding sites at positions -132 to $-125,-343$ to -336 , and -380 to -373 bp (depicted as F1, F2, and F3, respectively) from the transcriptional start site as predicted by MAPPER Search Engine. (B) Relative activation of FOXO1 and its mutants on various lengths of the native $H B P 1$ promoters. HEK-293T cells were co-transfected with luciferase reporters fused with the indicated lengths of the HBP1 promoter as well as the expression plasmid of wild-type FOXO1 or FOXO1 mutant (FOXO1AAA or FOXO1-H215R). Luciferase activity normalized to $\beta$-galactosidase was determined $24 \mathrm{~h}$ after transfection and represented as means \pm S.E.M. from three separate experiments. (C-D) A $0.5-\mathrm{kb} H B P 1$ promoter-luciferase construct with a series deletion combination of F1, F2 and/or F3 was co-transfected with a (C) FOXO1 or (D) FOXO1 or FOXO3a expression plasmid into HEK-293T cells. After $24 \mathrm{~h}$ of incubation, luciferase activity relative to control (empty vector) was determined after normalization to $\beta$-galactosidase. 
of FOXO1 to the human $H B P 1$ promoter in HSC-3 cells (Figure 5C). These data confirm that FOXO1 binds to the native human $H B P 1$ promoter in cellulo.

\section{FOXO1-mediated activation of HBP1 expression suppresses tumor cell proliferation and invasion}

Next, we examined whether FOXO1-mediated expression of HBP1 modulates tumorigenic growth and metastatic potential in oral cancer. As a downstream effector, HBP1 knockdown potently promoted cell malignancy as demonstrated by increased colony and invading cell numbers in HSC-3 cells (Figure 6A). Ectopic overexpression of constitutively active FOXO1 (FOXO1AAA) significantly suppressed colony growth in HSC-3 oral cancer cells; however, HBP1 knockdown alleviated the suppressive effect of FOXO1-AAA on colony formation (Figure 6B). To further examine the role of FOXO1mediated HBP1 expression in metastatic potential, both colony growth in soft agar and Matrigel invasion assay were performed in HSC-3 cells. Overexpression of either FOXO1 or HBP1 significantly decreased the ability of HSC-3 cells to form colonies in soft agar as compared with the vector control, while HBP1-specific siRNA diminished the suppressive effect of FOXO1 (Figure 6C).

A

\section{HBP1 promoter region}

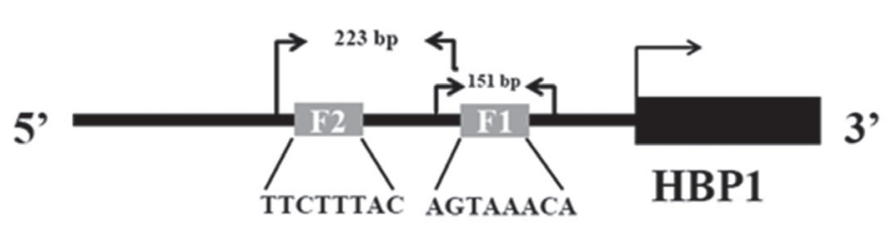

B

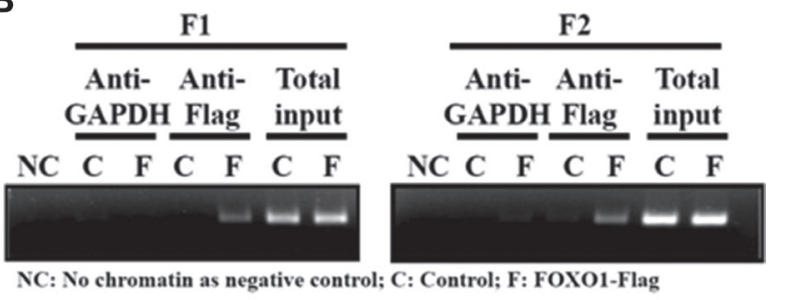

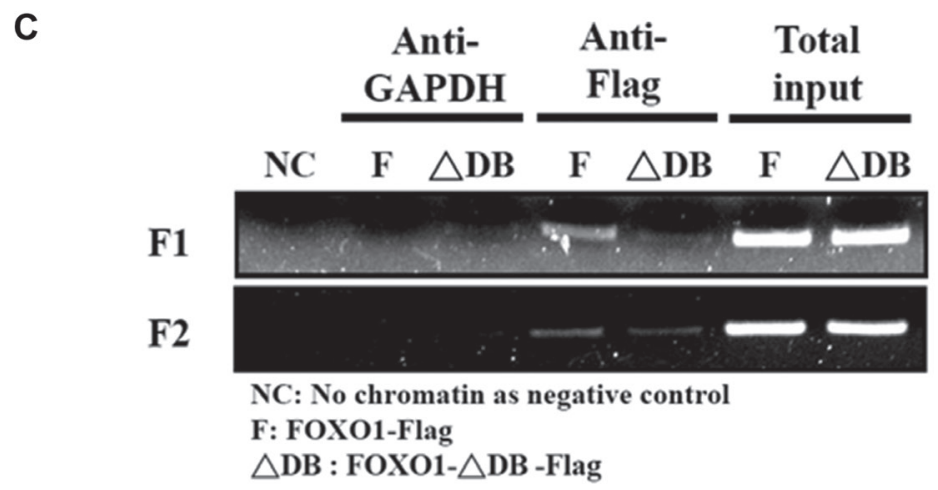

Figure 5: FOXO1 occupies its consensus binding sites in the endogenous HBP1 promoter. (A) A schematic diagram indicates two primer sets designed for the PCR detection and the expected sizes of the PCR products in the chromatin immunoprecipitation (ChIP) assay. (B-C) ChIP assay was performed to determine the formation of FOXO1-HBP1 promoter complex in (B) HEK-293T and (C) HSC3 cells. Cells were transfected with a pcDNA3 (Control; C), pcDNA3-FOXO1-Flag (FOXO1-Flag; F), or pcDNA3-FOXO1- $\triangle$ DB-Flag (FOXO1- $\triangle \mathrm{DB}$-Flag; $\triangle \mathrm{DB}$ ) plasmid for $48 \mathrm{~h}$, followed by sequential fixation, immunoprecipitation with anti-Flag or GAPDH antibody, and PCR analysis with the primer sets indicated in (A). 
A

\section{Control HBP1 shRNA}
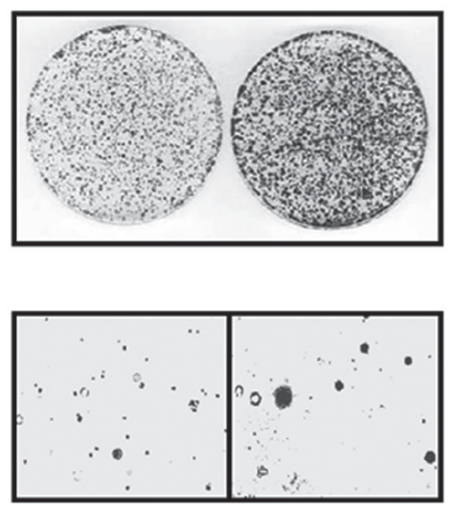

200X

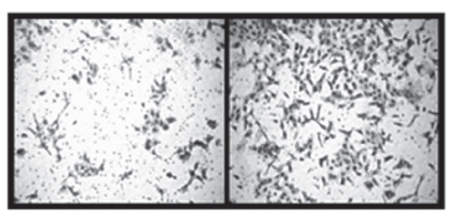

200X
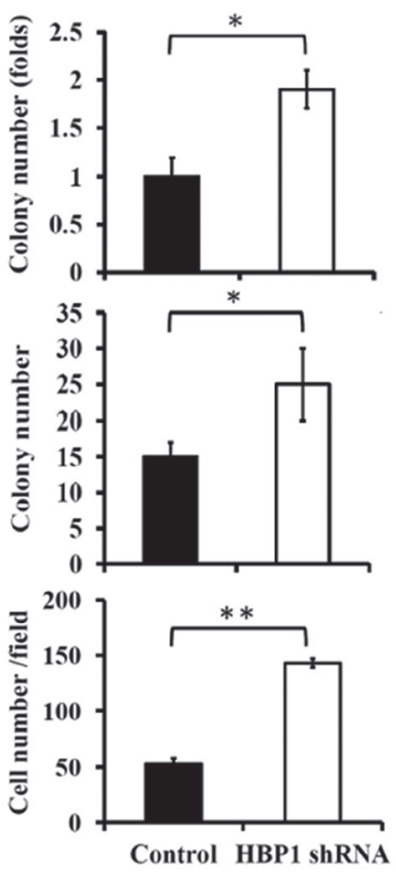

B

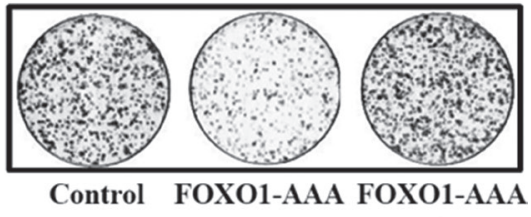

$+$

HBP1 siRNA

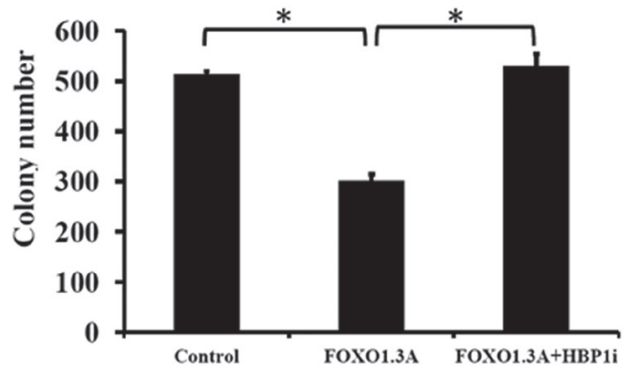

C
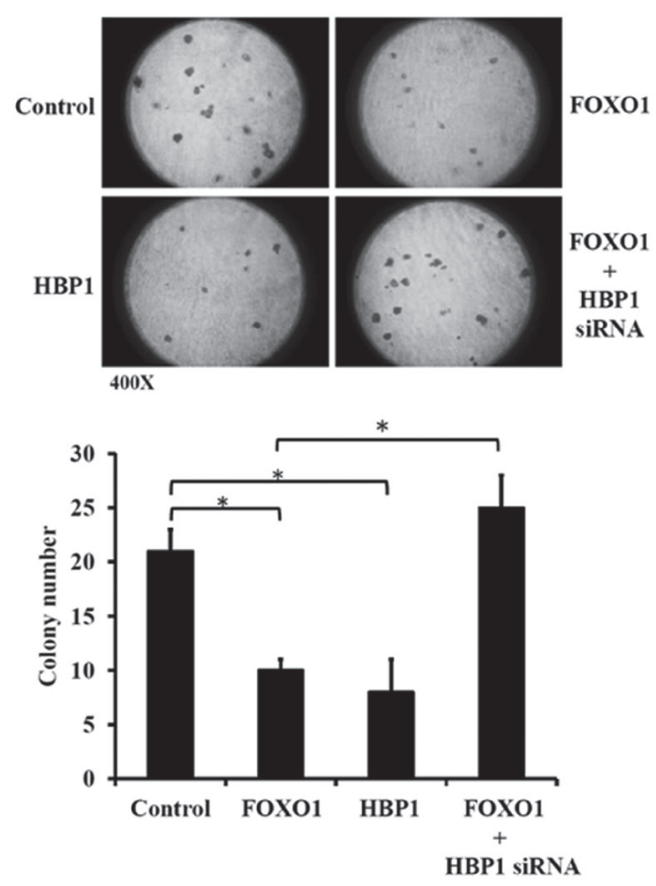

D
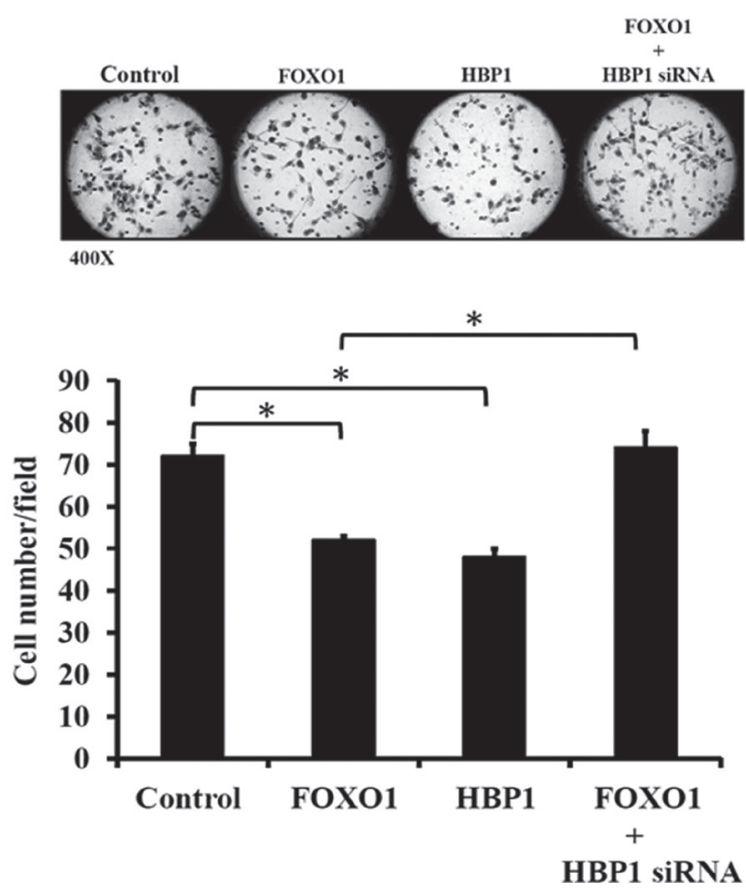

Figure 6: The role of FOXO1-mediated HBP1 expression in oral cancer malignancy. (A) HBP1 knockdown enhanced colony formation, anchorage-independent growth, and cellular invasion. HSC-3 cells were transfected with either pLKO or pLKO-HBP1 shRNA \#76 plasmid (Sigma) in $60 \mathrm{~mm}$ culture dishes, followed by assays for colony formation, anchorage-independent growth, and cellular invasiveness. Colony number or invading cells were quantified and ${ }^{*} p<0.05$ indicated a significant difference between the two designated groups. (B) Effect of the FOXO1-HBP1 axis on colony formation. HSC-3 cells transfected with FOXO1-AAA in the presence or absence of HBP1 siRNA were cultured in growth media for 7 days. Cell colonies were stained with crystal violet and the intensity was quantified at $540 \mathrm{~nm}\left({ }^{*} p<0.05\right.$ as compared with Control). (C-D) Effect of the FOXO1-HBP1 axis on invasive potential of oral cancer cells. HSC-3 cells $\left(3 \times 10^{4}\right)$ transfected with FOXO1-Flag (FOXO1), HBP1 cDNA (HBP1), or FOXO1-Flag along with either scramble siRNA or HBP1 siRNA (FOXO1 + HBP1 siRNA) were subjected to (C) colony formation in soft agar and (D) invasion assay as described in Materials and Methods. ${ }^{*} p<0.05$ indicated a significant difference between the two designated groups. 
and colleagues that the PI3K inhibitor LY294002 or other Akt inhibitor (Akt inhibitor VIII or MK 2206) caused increased mRNA or protein expression of HBP1 in either eosinophilic leukemia or colon carcinoma cells [18]. In addition, HBP1 expression coincided with the FOXO activity under regulation by Akt inhibitor GSK690693 [28]. Concomitant reduction of mRNA expression levels of FOXO1 with HBP1 were also observed in a set of breast tumors [18]. In the current study, we found a decreased FOXO1 mRNA level in invasive oral tumor specimen, and the combination of decreased mRNA expression levels of FOXO1 and HBP1 may predict invasiveness of oral cancer.

Through direct binding to the consensus binding sites in the promoter regions, the FOXO transcription factors regulate the transcription of their target genes [29]. The potential FOXO1 binding sites in the $H B P 1$ promoter were found -132 to $-125,-343$ to -336 , and -380 to -373 bp upstream of the HBP1 transcription start site (denoted as F1, F2, and F3, respectively) (Figure 4A) using the MAPPER Search Engine (http://genome.ufl.edu/mapper/). Employing reporter genes carrying different regions of the $H B P 1$ promoter, we found that a $0.5 \mathrm{~kb} H B P 1$ promoter, containing all three possible FOXO1 sites, displayed the strongest FOXO1 activation; however, a $0.2 \mathrm{~kb}$ promoter, which contains only one potential FOXO1 binding site, F1, was able to be stimulated by FOXO1, suggesting that this site is also a functional FOXO1 regulatory element (Figure 4B). Next we tested a series of single and combined deletions in the $0.5 \mathrm{~kb} \mathrm{HBP} 1$ promoter, and our results indicated that $\mathrm{F} 1$ and $\mathrm{F} 2$ are the major FOXO1 sites, while F3 is necessary for the full FOXO1 activation of the $H B P 1$ promoter (Figure 4C). Interestingly, FOXO1H215R, a FOXO1 mutant defect in the DNA binding domain, was able to activate the $H B P 1$ promoter although the induction was much weaker than that of the wild-type FOXO1 (Figure 4B), suggesting that HBP1 transcription can be regulated by FOXO1 through either direct or indirect binding. Indeed, FOXO1 can interact with other transcription factors to transcriptionally repress cyclin D1 (CCND1) and D2 (CCND2)[30] or activate cyclin G2 (CCNG2) and p130 (Rbl2) [31].

The HBP 1 promoter seems to respond preferentially to FOXO1 than FOXO3a. To differentiate the role of FOXO1 and FOXO3a in the activation of the HBP1 promoter, a series of reporter gene assays were employed. The results revealed that FOXO1 is a stronger activator than FOXO3a for either $2-\mathrm{kb}$ or $0.5-\mathrm{kb} H B P 1$ promoter (Figures 2E, 4D). Of special note, although both FOXO1 and FOXO3a are able to induce the $H B P 1$ promote activity, our data further indicate that the consensus binding site F2 is absolutely a prerequisite for the FOXO3a action on the $H B P 1$ promoter (Figure 4D), which is in an agreement with the finding by Coomans de Brachene et al. [18]. However, in the absence of F2, the binding site F1 still contributed to at least 2-fold of the FOXO1 activation on the HBP1 promoter (Figure 4B-4D). Taken together, our data support FOXO1 as a crucial upstream regulator of the $H B P 1$ gene transcription. Future study may reveal if the FOXO1-HBP1 axis and the FOXO3a-HBP1 axis share function redundancy, or one may be more active than the other under certain biological context.

Both FOXO1 and HBP1 are potential tumor suppressor genes; decreased FOXO1 expression [3] or loss-of-function $H B P 1$ mutation $[10,11]$ is often associated with tumorigenesis. FOXO1 and HBP1 may exert their tumor suppression function through the induction of growth arrest and apoptosis [4-6, 12, $14,15,23]$. Here, we showed that HBP1 can function as a downstream effector of FOXO1-mediated growth inhibition in oral cancer; HBP1 knockdown alleviated the suppressive effect of FOXO1 on colony formation (Figure 6B-6C). In addition, accumulated evidence also supports a role of FOXO1 and HBP1 in metastatic potential of cancer cells. Negative regulation of FOXO1 in cellular migration or invasion has been reported in various types of cancer, including prostate [32], and breast cancer [33]. Similarly, HBP1 knockdown hastens cell migration and invasion in breast and prostate cancer cells $[11,14]$. In the current study, we further provide evidence that the FOXO1-HBP1 axis may suppress the invasiveness of oral cancer cells (Figure 6D). Take together, the FOXO1HBP1 axis appears to be a potential therapeutic target of anticancer reagents in oral cancer.

\section{MATERIALS AND METHODS}

\section{Reagents and antibodies}

All chemicals were purchased from Sigma (St. Louis, MO) and antibodies were from Santa Cruz Biotechnology (Santa Cruz, CA), respectively, unless specified otherwise. Antibody for HBP1 was from Novus Biologicals (Littleton, $\mathrm{CO}$ ), and $\alpha$-tubulin was from Abcam (Cambridge, MA). PVDF membranes and ECL detection reagents for Western blotting analysis were purchased from Perkin Elmer Life Sciences, Inc. (Waltham, MA). Dual-light ${ }^{\circledR}$ system was from Applied Biosystems (Foster City, CA). PI3K inhibitor LY 294002 was purchased from Sigma (St. Louis, MO). Small interfering RNA (siRNA) molecules specific for HBP1 was purchased from Invitrogen (Carlsbad, CA).

\section{Plasmids}

Expressing plasmids, including pcDNA3-FlagFKHR(FOXO1)-Delta DB, pcDNA3-flag FKHRL1 (FOXO3a), pcDNA3-Flag-HA, pcDNA3-Flag-HA-Akt1, pcDNA3-Myr-HA-Akt1, and pcDNA3-T7-Akt1-T308AS473A [34] were purchased from Addgene (Cambridge, MA, USA). The HBP1 expressing plasmid pEF-BOS-HAHBP1, and FOXO1 expressing plasmids pcDNA3-A3FOXO1-Flag pcDNA3-WT-FOXO1-Flag and pcDNA3- 
H215R-FOXO1-Flag were kindly provided by Dr. Amy S. Yee and Dr. Brian Schaffhausen (Tufts University, USA), respectively.

\section{Cell culture and treatment}

For human oral squamous carcinoma cell lines, HSC3 and TW206 were kind gifts of Dr. Hsin-Ling Yang and SAS and OECM-1 cells were kindly provided by Dr. JangChang Lee at China Medical University (Taichung, Taiwan). HOK human oral keratinocytes and HEK (Homo sapiens embryonic kidney)-293T cells were obtained from Drs. Shieh Tzong-Ming and Tzong-Der Way at China Medical University, respectively (Taichung, Taiwan). HSC-3 and OECM-1 cells were maintained in Dulbecco's Modified Eagle Medium (DMEM)/F-12 and RPMI 1640 medium, and SAS, and 293T cells were maintained in DMEM, respectively, supplemented with $10 \%$ fetal bovine serum (FBS) and 1\% antibiotic-antimycotic. Cells were cultured in a $37^{\circ} \mathrm{C}, 5 \% \mathrm{CO}_{2}$ incubator. All cell culture reagents were from Invitrogen (Carlsbad, CA), unless indicated otherwise.

\section{Colony formation assay}

HSC-3 cells after transfection were cultured in growth media in $60 \mathrm{~mm}$ dishes for 7 days, and then washed with PBS, fixed with 10\% formalin (Mallinckrodt Chemicals, St. Louis, MO) for $10 \mathrm{~min}$, and stained with $0.05 \%$ crystal violet (Panreac Quimica S.A.U.) for $30 \mathrm{~min}$. Dishes were scanned for colony counting and crystal violet stain was dissolved in $100 \%$ methanol for optical density measurement at $540 \mathrm{~nm}$.

\section{Reporter assay}

The pGL3-luc plasmids containing 2037 (2 kb), or $526(0.5 \mathrm{~kb})$, or $166(0.2 \mathrm{~kb})$ bp HBP1 promoter and RSV- $\beta$-galactosidase plasmids were kindly provided by Dr. Amy S. Yee, Tufts University, USA. HEK-293T cells $\left(3 \times 10^{4}\right.$ cells $)$ cultured in a 24 -well plate were transfected with pDL3-HBP1-luc $(0.4 \mu \mathrm{g})$ and RSV- $\beta$ galactosidase $(0.1 \mu \mathrm{g})$ along with indicated vectors for $24 \mathrm{~h}$ and then cell lysates were collected in lysis solution (Applied Biosystems, Carlsbad, CA) according to the manufacturer's protocol. The Dual Light ${ }^{\circledR}$ System (Applied Biosystems) was used to quantify luciferase and $\beta$-galactosidase activities.

\section{Tissue sample preparation}

As previously described, [35] fresh oral tumor specimens were collected from oral cancer patients and stored in liquid nitrogen. Tumor tissues containing $>85 \%$ tumor cells based on the staining results were qualified for further RNA extractions. Acquisition of tissue specimens was approved by the institutional review board of the China Medical University Hospital. A total of 30 oral tumor specimens was obtained from the tissue bank at China Medical University Hospital (Taichung, Taiwan). Total RNA extracted from 9 normal oral epithelial counterparts was used as reference samples. All 30 tumor specimens were divided into two groups, non-invasive ( $n=18, \mathrm{p} N=0$, no metastasis) and invasive $(n=12$, $\mathrm{pN}>0$, lymph node metastasis).

\section{Reverse transcription-polymerase chain reaction, real-time $P C R$, and $P C R$}

Total RNA was extracted using RNeasy ${ }^{\circledR}$ Mini kit (Qiagen) according to the manufacturer's instructions. RT-PCR was performed using SuperScript ${ }^{\mathrm{TM}}$ III One-Step RT-PCR System with Platinum ${ }^{\circledR}$ Taq DNA Polymerase Kit (Invitrogen). The following primers were used: human HBP 1, 5'-ATCATCTCCTGTACACATCATAGC-3'(F) and 5'-CATAGAAAGGGTGGTCCAGCTTAC-3'(R); 18S, 5'-GTCTGTGATGCCCTTAGATG-3'(F) and 5'-AGCT TATGACCCGCACTTAC-3'(R). Primer sequences for real-time PCR analysis of oral tumor specimens were: HBP1, 5'-GAACCAATTCAGGCTCACA-3'(F) and 5'-TC AAGACTCAATGCTATCAGTATC-3'(R); FOXO1, 5'-AA GAGCGTGCCCTACTTCAA-3'(F) and 5'-CTGTTGTT GTCCATGGATGC-3'(R). Primer sequences for PCR analysis in ChIP assays were: $H B P 1-125,5^{\prime}$-TCTTTCG CCCTCTTATTGA-3' (F) and 5'-GAACTGCCATTCGG TTCTTC-3' (R); HBP1-336, F 5'-TTGTCCCAGACAC CAAAACA $-3^{\prime}(\mathrm{F})$ and 5'-GGATTGGACTATTGCCG AGA-3'(R).

\section{Matrigel invasion assay [36]}

Matrigel inserts for a 24-well chamber were purchased from BD Biosciences (Bedford, MA) and operated according to the manufacturer's protocol. HSC3 cell suspensions $\left(3 \times 10^{4}\right.$ cells $)$ after transfection with indicated plasmids were seeded onto the upper chamber in a serum-free medium and 10\% FBS-containing medium was added to the lower chamber to serve as a chemoattractant. After $24 \mathrm{~h}$ of incubation in a $37^{\circ} \mathrm{C}, 5 \%$ $\mathrm{CO}_{2}$ incubator, the non-invading cells from the upper chamber were removed using cotton swabs while the invading cells on the lower surface were fixed with $100 \%$ methanol, stained (Giesma in $20 \%$ ethanol), and counted. The invading cells were photographed and counted in five, randomly selected microscopic fields (200X magnification). Cell invasion was photographed under $400 \times$ magnification. Error bars in Figure $6 \mathrm{C}$ represent the variation of the cell numbers between the selected fields.

\section{Colony formation in soft agar [14]}

HSC -3 cells $\left(5 \times 10^{3}\right.$ cells $)$ after transfection with indicated constructs were suspended in $0.35 \%$ agar (DNA grade agarose, BIOMAN Scientific Co., Ltd, Taiwan) in DMEM containing 10\% FBS and plated in $35 \mathrm{~mm}$ Petri 
dishes with a $0.5 \%$ agar bottom layer. After 14 days of incubation at $37^{\circ} \mathrm{C}$ in a humidified incubator, the colonies $>200 \mu \mathrm{m}$ in diameter were counted microscopically within the field of a X40 objective lens (Olympus $1 \times 71$ ). Each bar represents the mean number of colonies $(\times 40$ field) \pm S.E.M. from three independent experiments.

\section{Chromatin immunoprecipitation (ChIP) assays}

Cells transfected with empty vector or vectors encoding FOXO1-Flag or FOXO1-Delta DB were subjected to chromatin immunoprecipitation experiments performed with the Chromatin Immunoprecipitation (ChIP) Assay Kit (Millipore), following the manufacturer's instructions. Anti-Flag and control anti-GAPDH antibody were purchased from Santa Cruz Biotechnology (Santa Cruz, CA) and DNA was extracted using DNA extraction kit (Favorgen biotech corp., Taiwan). PCR reactions were conducted using the Fermentas PCR kit (Thermo scientific, PA, USA), following the cycling conditions: 1 cycle $\left(95^{\circ} \mathrm{C}\right.$ $2 \mathrm{~min}), 30$ cycles $\left(95^{\circ} \mathrm{C} 30 \mathrm{~s}, 58^{\circ} \mathrm{C} 30 \mathrm{~s}, 72^{\circ} \mathrm{C} 1 \mathrm{~min}\right)$, and 1 cycle $\left(72^{\circ} \mathrm{C} 5 \mathrm{~min}\right)$ with the indicated primers. Migration of PCR products was performed in $2 \%$ agarose gels.

\section{Statistical analysis}

Data expressed as mean $\pm \mathrm{SD}$ or mean $\pm \mathrm{SEM}$ were calculated from at least three independent experiments. Statistical significance was analyzed using Student's $t$ test. Results were considered significantly different at $\mathrm{p}<0.05$.

\section{ACKNOWLEDGMENTS AND FUNDING}

The authors thank Dr. Amy S. Yee (Tufts University, USA) for the 2-kb and $0.5-\mathrm{kb} H B P 1$ promoter-luciferase reporter gene and $\beta$-galactosidase construct. This work was supported by the grants, NSC 98-2320-B-039-021MY3 from Ministry of Science and Technology, and CMU97-108 from China Medical University, Taiwan, R.O.C., to Chun-Yin Huang, and NSC102-2320-B-309001-MY3 from Ministry of Science and Technology, Taiwan, R.O.C. to Ming-Fen Lee.

\section{CONFLICTS OF INTEREST}

None declared.

\section{REFERENCES}

1. Greer EL, Brunet A. FOXO transcription factors at the interface between longevity and tumor suppression. Oncogene. 2005; 24:7410-7425.

2. Zhao Y, Wang Y, Zhu WG. Applications of post-translational modifications of FoxO family proteins in biological functions. J Mol Cell Biol. 2011.
3. Kim SY, Yoon J, Ko YS, Chang MS, Park JW, Lee HE, Kim MA, Kim JH, Kim WH, Lee BL. Constitutive phosphorylation of the FOXO1 transcription factor in gastric cancer cells correlates with microvessel area and the expressions of angiogenesis-related molecules. BMC Cancer. 2011; 11:264.

4. Katayama K, Nakamura A, Sugimoto Y, Tsuruo T, Fujita N. FOXO transcription factor-dependent p15(INK4b) and p19(INK4d) expression. Oncogene. 2008; 27:1677-1686.

5. Valis K, Prochazka L, Boura E, Chladova J, Obsil T, Rohlena J, Truksa J, Dong LF, Ralph SJ, Neuzil J. Hippo/ Mst1 stimulates transcription of the proapoptotic mediator NOXA in a FoxO1-dependent manner. Cancer Res. 2011; 71:946-954.

6. Suhara T, Kim HS, Kirshenbaum LA, Walsh K. Suppression of Akt signaling induces Fas ligand expression: involvement of caspase and Jun kinase activation in Akt-mediated Fas ligand regulation. Mol Cell Biol. 2002; 22:680-691.

7. Maiese K, Chong ZZ, Shang YC. OutFOXOing disease and disability: the therapeutic potential of targeting FoxO proteins. Trends Mol Med. 2008; 14:219-227.

8. Yee AS, Paulson EK, McDevitt MA, Rieger-Christ K, Summerhayes I, Berasi SP, Kim J, Huang CY, Zhang X. The HBP1 transcriptional repressor and the p38 MAP kinase: unlikely partners in G1 regulation and tumor suppression. Gene. 2004; 336:1-13.

9. Tevosian SG, Shih HH, Mendelson KG, Sheppard KA, Paulson KE, Yee AS. HBP1: a HMG box transcriptional repressor that is targeted by the retinoblastoma family. Genes Dev. 1997; 11:383-396.

10. Lin KM, Zhao WG, Bhatnagar J, Zhao WD, Lu JP, Simko S, Schueneman A, Austin GE. Cloning and expression of human HBP1, a high mobility group protein that enhances myeloperoxidase (MPO) promoter activity. Leukemia. 2001; 15:601-612.

11. Paulson KE, Rieger-Christ K, McDevitt MA, Kuperwasser C, Kim J, Unanue VE, Zhang X, Hu M, Ruthazer R, Berasi SP, Huang CY, Giri D, Kaufman S, et al. Alterations of the HBP1 transcriptional repressor are associated with invasive breast cancer. Cancer Res. 2007; 67:6136-6145.

12. Yao CJ, Works K, Romagnoli PA, Austin GE. Effects of overexpression of HBP1 upon growth and differentiation of leukemic myeloid cells. Leukemia. 2005; 19:1958-1968.

13. Kim J, Zhang X, Rieger-Christ KM, Summerhayes IC, Wazer DE, Paulson KE, Yee AS. Suppression of Wnt signaling by the green tea compound (-)-epigallocatechin 3-gallate (EGCG) in invasive breast cancer cells. Requirement of the transcriptional repressor HBP1. J Biol Chem. 2006; 281:10865-10875.

14. Chen YC, Zhang XW, Niu XH, Xin DQ, Zhao WP, Na YQ, Mao ZB. Macrophage migration inhibitory factor is a direct target of HBP1-mediated transcriptional repression that is overexpressed in prostate cancer. Oncogene. 2010; 29:3067-3078. 
15. Lee MF, Chan CY, Hung HC, Chou IT, Yee AS, Huang CY. $\mathrm{N}$-acetylcysteine (NAC) inhibits cell growth by mediating the EGFR/Akt/HMG box-containing protein 1 (HBP1) signaling pathway in invasive oral cancer. Oral Oncol. 2013; 49:129-135.

16. Shih HH, Tevosian SG, Yee AS. Regulation of differentiation by HBP1, a target of the retinoblastoma protein. Molecular and cellular biology. 1998; 18:4732-4743.

17. Berasi SP, Xiu M, Yee AS, Paulson KE. HBP1 repression of the p47phox gene: cell cycle regulation via the NADPH oxidase. Mol Cell Biol. 2004; 24:3011-3024.

18. Coomans de Brachene A, Bollaert E, Eijkelenboom A, de Rocca Serra A, van der Vos KE, Burgering BM, Coffer PJ, Essaghir A, Demoulin JB. The expression of the tumour suppressor HBP1 is down-regulated by growth factors via the PI3K/PKB/FOXO pathway. Biochem J. 2014; 460:25-34.

19. Brunet A, Bonni A, Zigmond MJ, Lin MZ, Juo P, Hu LS, Anderson MJ, Arden KC, Blenis J, Greenberg ME. Akt promotes cell survival by phosphorylating and inhibiting a Forkhead transcription factor. Cell. 1999; 96:857-868.

20. Obsil T, Obsilova V. Structure/function relationships underlying regulation of FOXO transcription factors. Oncogene. 2008; 27:2263-2275.

21. Ratushny V, Astsaturov I, Burtness BA, Golemis EA, Silverman JS. Targeting EGFR resistance networks in head and neck cancer. Cell Signal. 2009; 21:1255-1268.

22. Rubin Grandis J, Melhem MF, Barnes EL, Tweardy DJ. Quantitative immunohistochemical analysis of transforming growth factor-alpha and epidermal growth factor receptor in patients with squamous cell carcinoma of the head and neck. Cancer. 1996; 78:1284-1292.

23. Huang CY, Chan CY, Chou IT, Lien CH, Hung HC, Lee MF. Quercetin induces growth arrest through activation of FOXO1 transcription factor in EGFR-overexpressing oral cancer cells. J Nutr Biochem. 2013; 24:1596-1603.

24. Armoni M, Harel C, Karni S, Chen H, Bar-Yoseph F, Ver MR, Quon MJ, Karnieli E. FOXO1 represses peroxisome proliferator-activated receptor-gammal and -gamma2 gene promoters in primary adipocytes. A novel paradigm to increase insulin sensitivity. J Biol Chem. 2006; 281:19881-19891.

25. Huang H, Tindall DJ. Dynamic FoxO transcription factors. Journal of cell science. 2007; 120:2479-2487.

26. Vivanco I, Sawyers CL. The phosphatidylinositol 3-Kinase AKT pathway in human cancer. Nat Rev Cancer. 2002; 2:489-501.
27. Courtney KD, Corcoran RB, Engelman JA. The PI3K pathway as drug target in human cancer. J Clin Oncol. 2010; 28:1075-1083.

28. Kumar R, Blakemore SJ, Ellis CE, Petricoin EF, 3rd, Pratt D, Macoritto M, Matthews AL, Loureiro JJ, Elliston K. Causal reasoning identifies mechanisms of sensitivity for a novel AKT kinase inhibitor, GSK690693. BMC Genomics. 2010; 11:419.

29. Kaestner KH, Knochel W, Martinez DE. Unified nomenclature for the winged helix/forkhead transcription factors. Genes Dev. 2000; 14:142-146.

30. Ramaswamy S, Nakamura N, Sansal I, Bergeron L, Sellers WR. A novel mechanism of gene regulation and tumor suppression by the transcription factor FKHR. Cancer Cell. 2002; 2:81-91.

31. Chen J, Yusuf I, Andersen HM, Fruman DA. FOXO transcription factors cooperate with delta EF1 to activate growth suppressive genes in B lymphocytes. J Immunol. 2006; 176:2711-2721.

32. Zhang H, Pan Y, Zheng L, Choe C, Lindgren B, Jensen ED, Westendorf JJ, Cheng L, Huang H. FOXO1 inhibits Runx2 transcriptional activity and prostate cancer cell migration and invasion. Cancer Res. 2011; 71:3257-3267.

33. Wu Y, Elshimali Y, Sarkissyan M, Mohamed H, Clayton S, Vadgama JV. Expression of FOXO1 is associated with GATA3 and Annexin-1 and predicts disease-free survival in breast cancer. Am J Cancer Res. 2012; 2:104-115.

34. Ramaswamy S, Nakamura N, Vazquez F, Batt DB, Perera S, Roberts TM, Sellers WR. Regulation of G1 progression by the PTEN tumor suppressor protein is linked to inhibition of the phosphatidylinositol 3-kinase/Akt pathway. Proc Natl Acad Sci USA. 1999; 96:2110-2115.

35. Sheu JJ, Hua CH, Wan L, Lin YJ, Lai MT, Tseng HC, Jinawath N, Tsai MH, Chang NW, Lin CF, Lin CC, Hsieh LJ, Wang TL, et al. Functional genomic analysis identified epidermal growth factor receptor activation as the most common genetic event in oral squamous cell carcinoma. Cancer research. 2009; 69:2568-2576.

36. Huang CY, Chou YH, Hsieh NT, Chen HH, Lee MF. MED28 regulates MEK1-dependent cellular migration in human breast cancer cells. J Cell Physiol. 2012; 227:3820-3827. 